\title{
NGHIÊN CÚU CÁC YẾU TỐ ẢNH HƯởNG ĐẾN Độ LOE MỐI HÀN CỦA MÁY UỐN, HÀN VÒNG THÉP DÂY TỰ ĐộNG
}

\author{
CHÂU MINH QUANG, NGUYẼ̃N ĐỨC TRƯỜNG, CHÂU CAO NGUYÊN KHANG \\ Truờng Đại học Công nghiệp Thành phố Hồ Chí Minh; \\ chauminhquang@iuh.edu.vn
}

Tóm tắt. Trong những năm gần đây, vòng thép dây được sử dụng ngày càng rộng rãi. Các nhà máy, xí nghiệp sản xuất vòng thép dây đã áp dụng phương pháp hàn tiếp điểm để gia công sản phẩm này, nhưng chất lượng mối hàn và năng suất thấp.

Bài báo này tập trung vào việc nghiên cứu các yếu tố ảnh hưởng chính đến độ loe mối hàn trên máy uốn, hàn vòng thép dây tự động. Nghiên cứu thực nghiệm và tìm được phương trình hồi quy mô tả ảnh hươong của các thông số liên quan. Kết quả của quá trình nghiên cứu được thể hiện ở phần 3 trong bài báo này.

Từ khóa: máy hàn vòng, máy uốn vòng và máy uốn, hàn vòng thép dây tụ động

\section{RESEARCH THE FACTORS AFFECTING THE REDUNDANCY WELD MACHINES WELDING OF STEEL COIL WIRE AUTOMATIC}

\begin{abstract}
In recent years, steel wire ring is used widely. Many companies, which in making steel wire ring field, used fusing of contact method to make products. But welding quality and low performance.

This paper proposes studying the factors affecting the redundancy weld machines welding of steel coil wire automatic, experimental research, find recursive equation, to describe the affect of relate parameters. The results of this research have part 3
\end{abstract}

Key words: Steel wire ring, bending ring machine and bending, welding wire ring machine steel coil automatic.

\section{GIỚI THIỆU}

Trong những năm gần đây, rất nhiều doanh nghiệp trong nước gặp khó khăn trong sản xuất vòng thép vì sản xuất thủ công cho năng suất và chất lượng thấp. Phương pháp hàn điện tiếp xúc, tự động hóa khi hàn được các nhà khoa học trong và ngoài nước đặc biệt quan tâm. M. Pouranvari và $S$. $P$. H. Marashi [1] đã nghiên cứu các yếu tố ảnh hưởng đến tính chất cơ học của mối hàn điểm tiếp xúc và chỉ ra mối tương quan giữa các thông số như cường độ dòng hàn, thời gian hàn, lực ép hàn, phân tích các đặc tính vật lý về vị trí hàn và đặc tính cơ học. Sử dụng tải trọng điểm và hấp thụ năng lượng của mối hàn tại điểm tiếp xúc trong thời gian thí nghiệm cắt và kéo nén để mô tả tính chất mối hàn tiếp điểm. Thấy rằng kích thước mối hàn, chế độ phá hủy và độ bền, độ dẻo của điểm hư hỏng là những yếu tố chính ảnh hưởng đến tải trọng đỉnh và hấp thụ năng lượng của mối hàn tiếp điểm. M. Zhou [2] nghiên cứu các yếu tố ảnh hưởng để nâng cao hiệu suất và chất lượng cho các tính chất của mối hàn. Trong nghiên cứu của L. Boriwal, R. M. Sarviya, $M . M$. Mahapatra [3] về việc mô phỏng logic mờ của quá trình hàn tiếp điểm để dự đoán độ bền của mối hàn. Nghiên cứu cho thấy rằng, dòng điện, thời gian và lực ép hàn được coi là các yếu tố ảnh hưởng chính của quá trình. Anna Runnemalm, et al [4] đã đưa ra phương pháp kiểm tra bằng nhiệt. Hệ thống sử dụng ánh sáng làm nguồn kích thích có thể phát hiện các mối hàn điểm và đo đường kính mối hàn thông qua một thuật toán. P. Puschner [5] and Satoshi NAKAMURA [6] đã nghiên cứu tự động hóa khi hàn. Tuy nhiên các nghiên cứu trên vẫn chưa đề cập đến chất lượng và năng suất sản phẩm vòng thép, do đó bài báo này đưa ra kết quả nghiên cứu khi hàn vòng thép trên máy hàn tự động. 


\section{THIẾT KẾ MÔ HÌNH MÁY UỐN, HÀN VÒNG THÉP DÂY TỰ ĐộNG}

Hiện nay, các công ty, xí nghiệp ngày càng mở rộng sản xuất, kinh doanh. Thực tế yêu cầu sử dụng máy tự động hóa trong việc hàn vòng thép dây để nâng cao năng suất và chất lượng sản phẩm cũng như giảm công lao động. Việc sử dụng dây chuyền sản xuất tự động tiên tiến mang lại nhiều hiệu quả, phù hợp với quy mô, trình độ sản xuất của xí nghiệp.

Nhận thấy cách làm vòng thép dây bằng máy uốn, hàn vòng thủ công cho năng suất, chất lượng sản phẩm không ổn định, độ chính xác không cao như hình $1 \mathrm{c}$. Từ đó, làm tăng chi phí sản xuất và giá thành sản phẩm

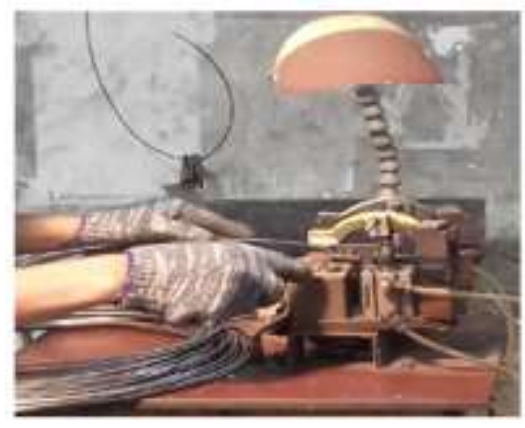

Hinh a

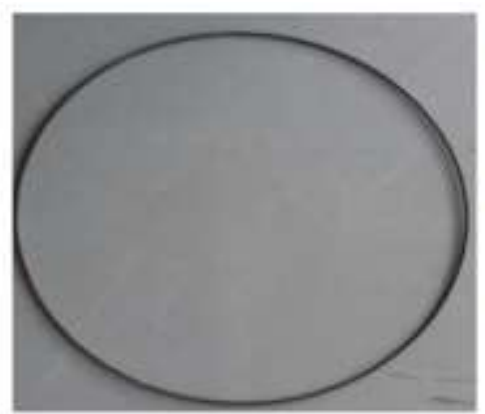

Hinh b

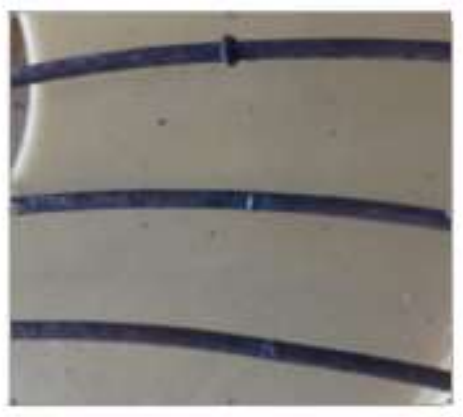

Hinh c

Hình 1. Máy hàn thủ công truyền thống và sản phẩm

a) Hàn thủ công; b) Vòng thép dây; c) Kiểu độ loe mối hàn

Qua quá trình uốn và cắt thì vòng thép dây được hàn bằng máy hàn thủ công như hình $1 \mathrm{a}$. Sản phẩm vòng thép dây như hình $1 \mathrm{~b}$ ở mối hàn ghép nối có độ loe không ổn định về đường kính mối hàn ở hình $1 \mathrm{c}$ gây hư hỏng hoặc khó gia công ở các bước tiếp theo của quá trình sản suất.

Để khẳc phục những vấn đề nêu trên, ta cần phải có một cơ cấu làm việc với các thông số phù hợp. Chính vì vậy, để đáp ứng những yêu cầu trên, ta phải tiến hành quá trình nghiên cứu thực nghiệm nhằm tìm ra các thông số kỹ thuật phù hợp nhất của máy uốn, hàn vòng thép dây tự động để nâng cao năng suất và chất lượng sản phẩm, tăng khả năng cạnh tranh và đáp ứng các yêu cầu cao nhất cho sản phẩm của thị trường trong nước và quốc tế.

Vật liệu: thép dây dạng cuộn CT3, đường kính 3,15mm

Thiết bị: máy uốn, hàn vòng thép dây tự động

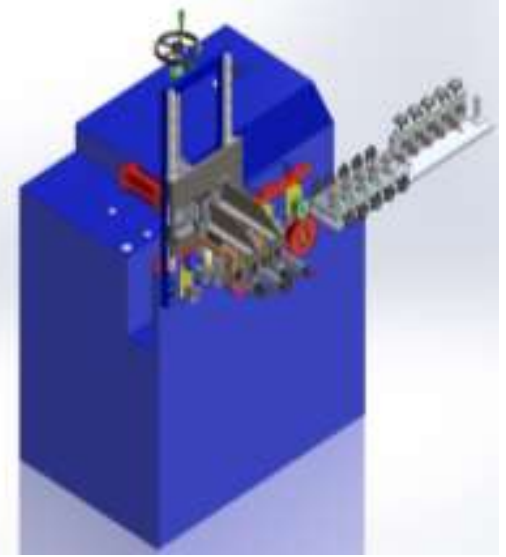

a

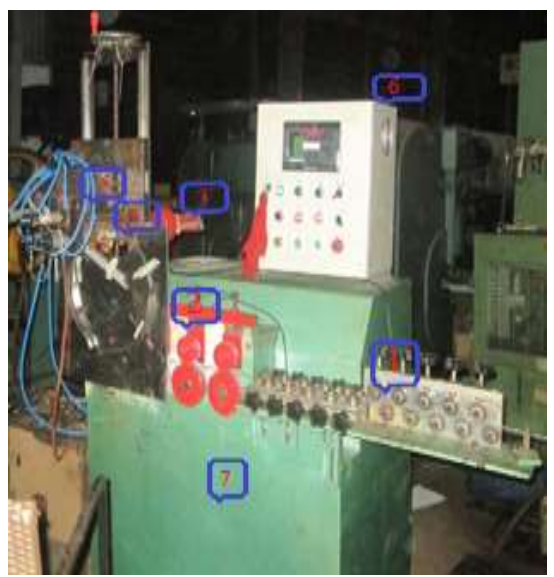

$b$

Hình 2. Mô hình 3D và thực tế máy uốn, hàn vòng thép dây tự động

2a) Mô hình thiết kế 3D; 2b) Máy uốn, hàn vòng thép dây tự động. 
Mô hình thiết kế 3D của máy uốn hàn vòng thép dây tự động như hình 2a. Dựa trên mô hình thiết kế, qua quá trình gia công, chế tạo và lắp ráp thành công máy hàn vòng thép dây thể hiện ở hình $2 b$. Cơ cấu của máy bao gồm các cụm chính sau. 1- Bộ con lăn nắn thẳng để nắn thẳng dây thép từ cuộn cấp; 2- Bộ con lăn uốn để uốn vòng thép dây theo bán kính cho trước; 3- Dao cắt vòng dây khi đủ chu vi vòng thép dây; 4- Máy hàn nối hai đầu vòng thép dây; 5- Kẹp hàn giữ chặt 2 đầu vòn thép dây; 6- Tủ điều khiển; 7- Khung máy

Nguyên lý hoạt động của máy uốn, hàn vòng thép dây tự động được thể hiện như hình 3 . Phôi thép dây ở dạng cuộn thông qua bộ phận dẫn hướng được đưa vào bộ con lăn nắn thẳng dây thép (1). Tại đây bộ con lăn nắn thẳng được nối với động cơ servor tạo moment quay đưa thép dây đến bộ con lăn uốn (2). Khi uốn đủ độ dài yêu cầu, định vị thép dây thực hiện thao tác cắt dây thép (3). Lúc này, cơ cấu kẹp (5) thực hiện thao tác kẹp chặt 2 đầu tự do của dây thép sát lại nhau và thực hiện thao tác hàn tự động (4). Khi kết thúc quá trình hàn, cơ cấu kẹp nhã kẹp để thả vòng thép dây xuống khay và đồng thời rút về và lặp lại quá trình trên.

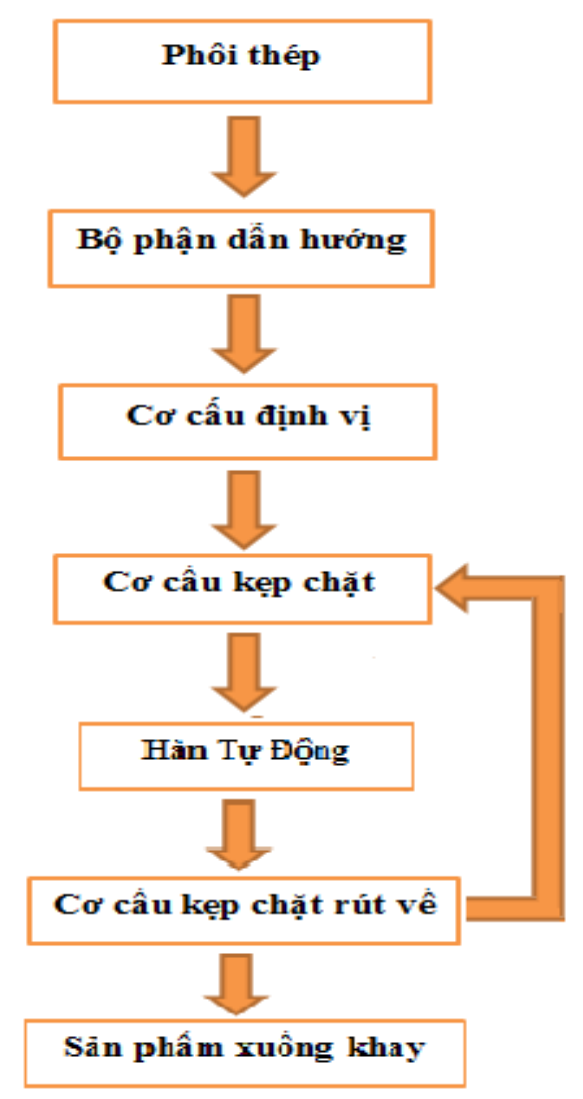

Hình 3. Sơ đồ nguyên lý hoạt động của máy uốn, hàn vòng thép dây tự động

\section{KẾT QUẢ NGHIÊN CỨU VÀ THẢO LUẬN}

\subsection{Kết quả bài toán quy hoạch thực nghiệm.}

Nội dung nghiên cứu nhằm mục đích xác định ảnh hưởng của các thông số về điện, cơ khí đến các chỉ tiêu về yêu cầu kỹ thuật của máy và mối quan hệ giữa chúng. Do máy có nhiều biến số thay đổi với nhiều mức khác nhau, muốn giảm số lượng thí nghiệm mà vẫn đảm bảo độ chính xác của thí nghiệm. Từ đó xác định các thông số hoạt động hợp lý của máy.

Sau khi tiến hành loại bỏ các yếu tố ít ảnh hưởng. Ta chọn được 3 yếu tố ảnh hưởng chính đó là lực ép, dòng diện và thời gian hàn với khoảng biến thiên giá trị được trình bày ở bảng 1 . Lực ép hàn dao động từ 2.5 đến $3.5 \mathrm{~Pa}$, dòng điện hàn dao động từ 18 đến $22 \mathrm{~A}$ (ở mức 8 đến 10 của biến áp sử dụng trong thí nghiệm), thời gian hàn dao động từ 5 đến $7 \mathrm{~ms}$. 
Bảng 1. Các giá trị ảnh hưởng đến độ loe mối hàn

\begin{tabular}{|l|c|c|l|}
\hline \multicolumn{1}{|c|}{ Đối tượng } & Thấp nhất & Cao nhất & Đơn vị \\
\hline Lực ép hàn & 2.5 & 3.5 & $\mathrm{~Pa}$ \\
\hline Dòng điện hàn & 18 & 22 & $\mathrm{~A}$ \\
\hline Thời gian hàn & 5 & 7 & $\mathrm{~ms}$ \\
\hline
\end{tabular}

\subsection{Kết quả thực nghiệm và xử lý kết quả thực nghiệm.}

Ma trận thí nghiệm được lập ngẫu nhiên bằng chương trình xử lý số liệu thực nghiệm. Thông số đầu ra: độ loe của mối hàn, kí hiệu là $\mathrm{Y}$, đây là thông số đặc trưng cho mục đích nghiên cứu. Đại lượng này chịu ảnh hưởng của hàng loạt thông số đầu vào và nhiễu. Độ loe mối hàn được đo trực tiếp bằng thước kẹp điện tử.

Giá trị trung bình của độ loe mối hàn được tính bằng công thức sau (1)

$$
m_{x}=\bar{x}=\frac{\sum_{i=1}^{N} x_{i}}{N}
$$

$\mathrm{X}_{\mathrm{i}}$ - Số đo đại lượng ngẫu nhiên $\mathrm{x}$ ở thí nghiệm thứ $\mathrm{i}$

$\mathrm{N}$ - Số lượng mẫu

Tiến hành thí nghiệm thực nghiệm, ở mỗi thí nghiệm tiến hành lấy 50 mẫu ứng với 15 lần thay đổi thông số. Với mỗi thí nghiệm thực hiện đo và lấy giá trị trung bình ta được kết quả ở bảng 2 .

Bảng 2. Kết quả thí nghiệm

\begin{tabular}{|c|c|c|c|c|}
\hline STT & Lực ép hàn $(\mathrm{Pa})$ & Dòng điện hàn $(\mathrm{A})$ & Thời gian hàn $(\mathrm{ms})$ & Độ loe trung bình $(\mathrm{mm})$ \\
\hline 1 & 2.5 & 20 & 6 & 3.27 \\
\hline 2 & 2.5 & 22 & 5 & 3.296 \\
\hline 3 & 2.5 & 22 & 7 & 3.322 \\
\hline 4 & 2.5 & 18 & 7 & 3.267 \\
\hline 5 & 2.5 & 18 & 5 & 3.16 \\
\hline 6 & 3 & 20 & 5 & 3.234 \\
\hline 7 & 3 & 18 & 6 & 3.279 \\
\hline 8 & 3 & 20 & 6 & 3.296 \\
\hline 9 & 3 & 22 & 6 & 3.45 \\
\hline 10 & 3 & 20 & 7 & 3.34 \\
\hline 11 & 3.5 & 18 & 5 & 3.19 \\
\hline 12 & 3.5 & 22 & 5 & 3.34 \\
\hline 13 & 3.5 & 20 & 6 & 3.33 \\
\hline 14 & 3.5 & 18 & 7 & 3.291 \\
\hline 15 & 3.5 & 22 & 7 & 3.58 \\
\hline
\end{tabular}

Số liệu thu được sau khi phân tích tính toán được đưa vào ma trận thí nghiệm để làm dữ liệu tính các hệ số của mô hình. Qua quá trình sử lý số liệu thu được phương trình hồi quy của từng yếu tố và tổng 3 yếu tố ảnh hưởng đến độ loe mối hàn như phương trình (3.1), (3.2), (3.3) và (3.4)

Với Y: Độ loe của mối hàn; X1: lực ép hàn, X2: thời gian hàn, X3: dòng điện hàn

\subsection{1 Ảnh hưởng của lực ép lên mối hàn.}

Lực ép giữa 2 đầu tác dụng lên mối hàn có mức ảnh hưởng tuyến tính theo độ lớn tác dụng và được biểu diển như hình 4 . Với R-squared $=100 \%$. Sự biến đổi được mô tả mối quan hệ giữa lực ép và độ loe của mối hàn dựa trên phương trình đa thức bậc 2 sau: 


$$
\mathrm{Y}=2.654+0.161 * \mathrm{X} 2-0.009 * \mathrm{X} 2 \wedge 2(3.1)
$$

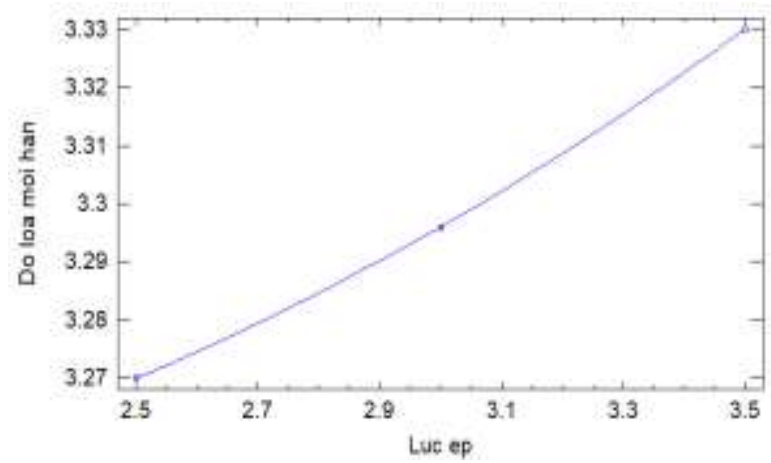

Hình 4. Ảnh hưởng của lực ép đến độ loe mối hàn

\subsubsection{Anh hưởng của thời gian lên mối hàn.}

Lực ép hàn ảnh hưởng tuyến tính đến độ loe mối hàn được biểu diển ở hình 5 với

Với R-squared $=100 \%$. Sự biến đổi được mô tả mối quan hệ giữa thời gian đối với độ loe của mối hàn dựa trên phương trình đa thức bậc 2 sau:

$\mathrm{Y}=2.654+0.161 * \mathrm{X} 2-0.009 * \mathrm{X} 2^{\wedge} 2(3.2)$

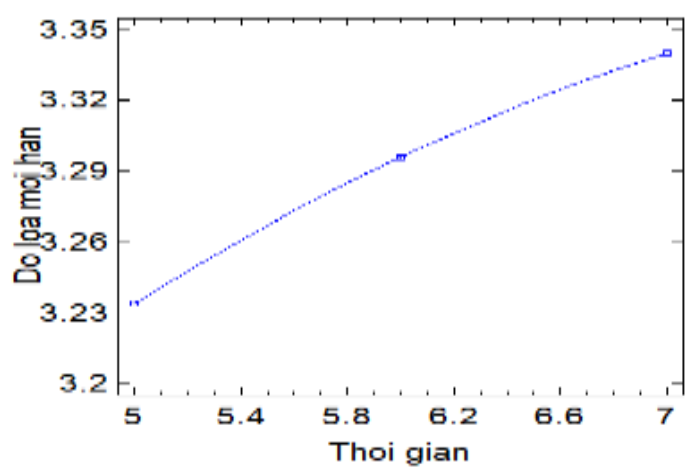

Hình 5. Ảnh hưởng của thời gian đến độ loe mối hàn

\subsubsection{Anh hưởng của dòng điện lên mối hàn.}

6

Dòng điện hàn ảnh hưởng tuyến tính đến độ loe mối hàn với mức ổn định cao được biểu diển ở hình

Với R-squared $=100 \%$. Sự biến đổi mô tả bởi mối quan hệ giữa lực ép và độ loe của mối hàn dựa trên phương trình đa thức bậc 2 sau: như hình 6 


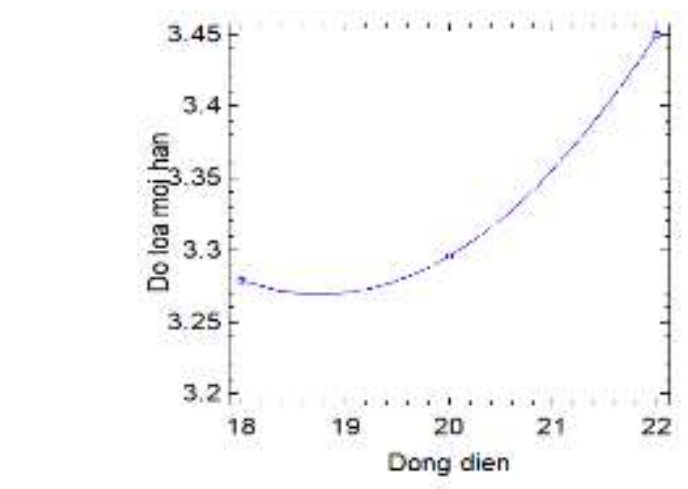

$\mathrm{Y}=9.291-0.64225^{*} \mathrm{X} 3+0.017125^{*} \mathrm{X} 3^{\wedge} 2$

Hình 6. Ảnh hưởng của dòng diện đến độ loe mối hàn

\subsection{4 Ảnh hưởng của lực ép, dòng điện, thời gian lên mối hàn.}

Tiến hành phân tích phương sai kết quả thực nghiệm cho thấy đối với hàm mô tả độ loe của mối hàn $\mathrm{Y}$ bởi các yếu tố ảnh hưởng $\mathrm{X} 1, \mathrm{X} 2, \mathrm{X} 3$ có các hệ số hồi quy đều đảm bảo độ tin cậy. Mức ý nghĩa $\mathrm{R}$ - squared là $80.6733 \%$ với sai số độ lệch chuẩn

Kết quả tính toán mô hình toán ở dạng thực ta có hàm số mô tả độ loe $(\mathrm{mm})$ :

$$
\begin{aligned}
& \mathrm{Y}=7.70862-0.371467 * \mathrm{X} 1-0.520811 * \mathrm{X} 3+0.224167 * \mathrm{X} 2-0.0795556 * \mathrm{X} 1{ }^{\wedge} 2+0.031 * \mathrm{X} 1 * \mathrm{X} 3 \\
& +0.052 * \mathrm{X} 1 * \mathrm{X} 2+0.0111528 * \mathrm{X} 3 \wedge 2+0.003625 * \mathrm{X} 3 * \mathrm{X} 2-0.0328889 * \mathrm{X} 2{ }^{\wedge} 2 ; \text { (3.4) }
\end{aligned}
$$

Bài toán tối ưu hóa được xây dựng trên cơ sở các hàm toán là các phương trình hồi quy xác định bằng phương pháp quy hoạch thực nghiệm có dạng đa thức bậc II. Phương trình (3.4) xác định hàm mục tiêu với các thông số đặc trưng cho mục đích nghiên cứu.

Bài toán tối ưu hóa được xây dựng trên cơ sở các hàm toán là các phương trình hồi quy xác định bằng phương pháp quy hoạch thực nghiệm có dạng đa thức bậc II. Phương trình (3.4) xác định hàm mục tiêu với các thông số đặc trưng cho mục đích nghiên cứu.

Phân tích mô hình thống kê thực nghiệm:

Mức độ ảnh hưởng của các thông số được biểu diển bằng các biểu đồ thể hiện trên hình 7 và hình 8 với các thông số ở dạng thực.

Biểu đồ này được vẽ khi các yếu tố khác được giữ cố định.

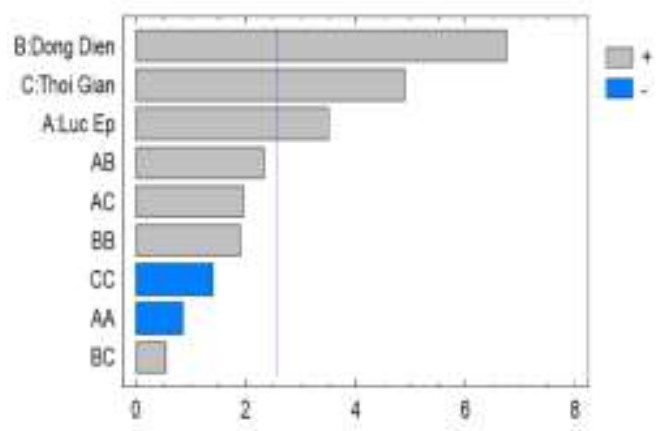

Hình 7. Biểu đồ thể hiện ảnh hưởng của các thông số đến độ loe mối hàn ở dạng thực. 
Căn cứ vào biểu đồ hình 7 cho thấy các yếu tố đầu vào của nghiên cứu ảnh hưởng đến độ loe mối hàn ở mức khác nhau, mức độ ảnh hưởng đến độ loe mối hàn sắp xếp giảm dần theo thứ tự: thời gian, dòng điện, lực ép khi hàn. Các yếu tố ảnh hưởng đều có độ ảnh hưởng tuyến tính với độ loe của mối hàn thể hiện trên hình $8 \mathrm{a}$ và $8 \mathrm{~b}$. Khi thay đổi bất kì 1 trong 3 yếu tố làm thí nghiệm thì độ loe của mối hàn cũng tăng đều lên.

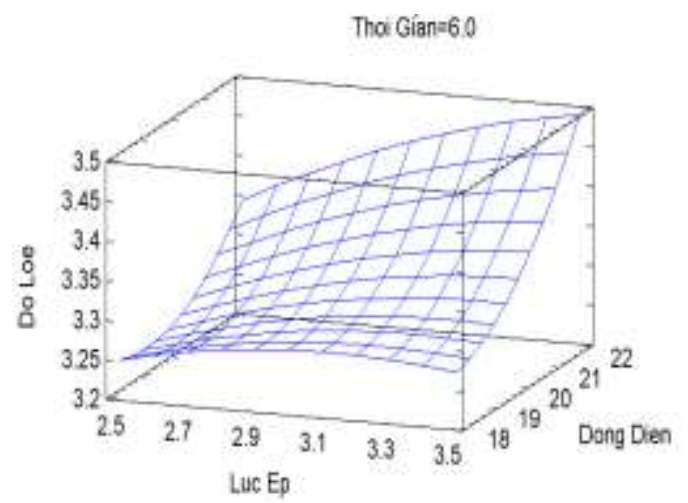

Hình 8a

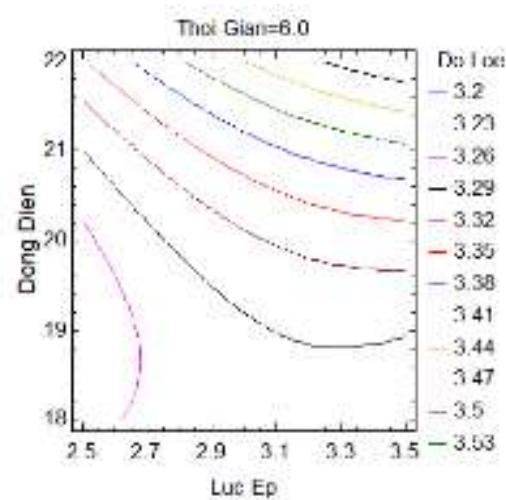

Hình 8b

Hình $8 \mathrm{a}, 8 \mathrm{~b}$. Mức độ ảnh hưởng của các yếu tố đến độ loe mối hàn Y

\subsection{Năng suất}

\subsubsection{So sánh máy truyền thống và máy hàn vòng thép dây tự động sau cải tiến}

Cho 3 máy uốn hàn vòng tự động hoạt động cùng lúc với 3 dây chuyền máy uốn và hàn vòng truyền thống trong 1 ca làm việc 8 giờ ta thu được kết quả như ở bảng 3 .

Bảng 3. Bảng so sánh giữa máy truyền thống và máy uốn, hàn vòng thép dây tự động sau cải tiến

\begin{tabular}{|c|c|c|c|}
\hline & Nguời vận hành & Năng suất (vòng) & Năng lượng tiêu thụ $(\mathrm{KW})$ \\
\hline Thủ công & 7 & 2400 & 50 \\
\hline Tự động & 2 & 4000 & 40 \\
\hline
\end{tabular}

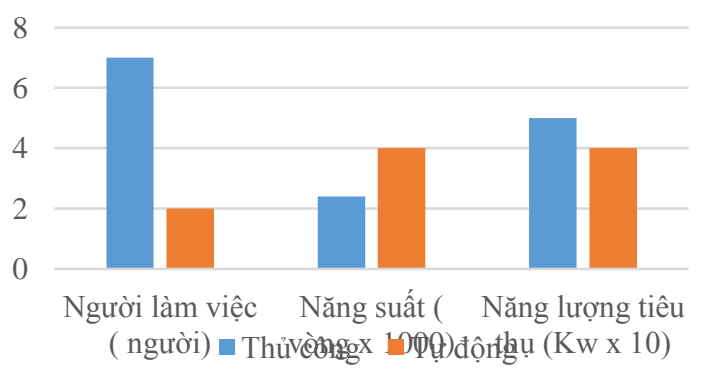

Hình 9. So sánh giữa phương pháp truyền thống và máy tự động sau cải tiến

Từ số liệu ở bảng 3 vẽ được biểu đồ ở hình 9, năng suất làm việc của dây chuyền sản suất truyền thống và máy tự động sau cải tiến cho thấy máy tự động thì máy tự động đạt năng suất cao cao hơn. Khi cho hoạt 
động 3 dây chuyền chuyền truyền thống và 3 máy uốn hàn vòng tự động thì máy tự động cho năng suất gấp 5,8 lần so với máy truyền thống với mức tiêu thụ năng lượng giảm $20 \%$

\subsubsection{So sánh máy hàn vòng thép dây cũ và máy sau cải tiến}

Cho máy uốn, hàn vòng thép dây tự động cũ hoạt động cùng lúc với máy sau cải tiến trong 1 ca làm việc 8 giờ ta thu được kết quả như ở bảng 4 :

Bảng 4. So sánh máy hàn vòng thép dây cũ và máy sau cải tiến

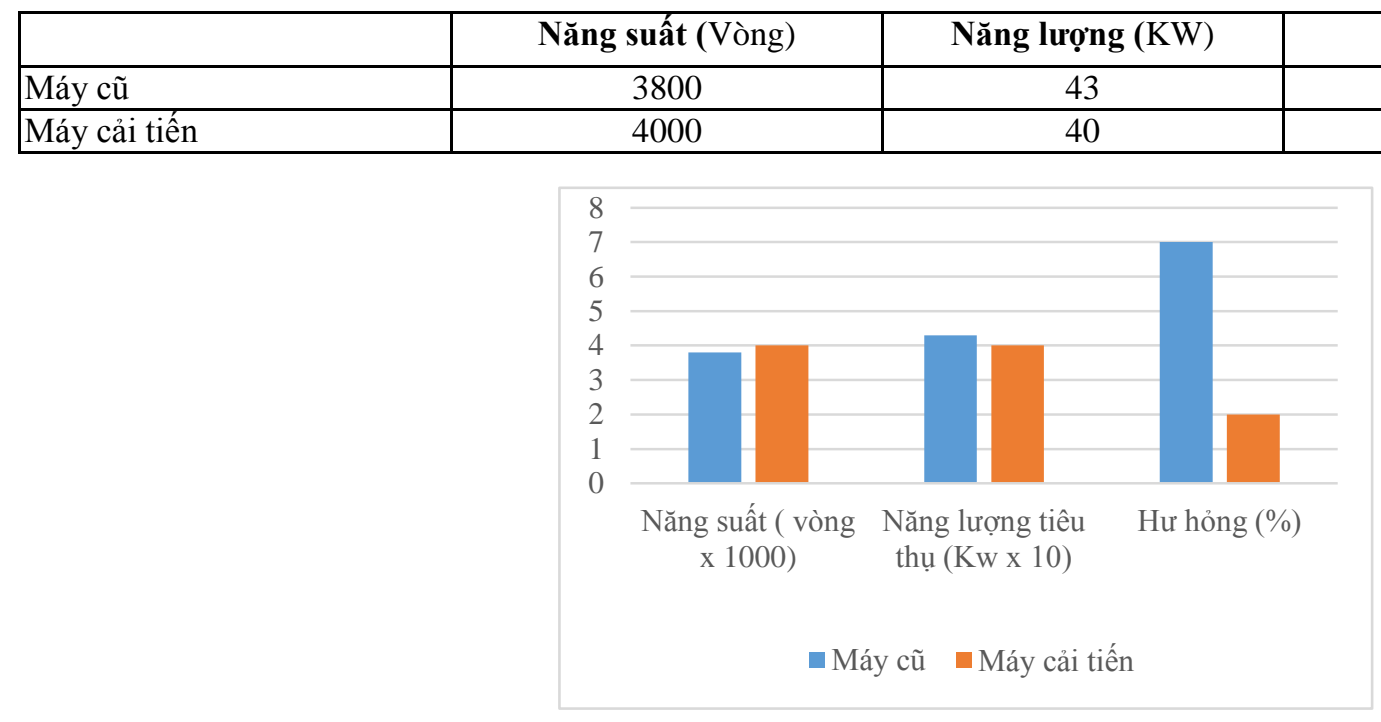

Hình 10. So sánh máy uốn, hàn vòng thép dây cũ và máy sau cải tiến

Thông qua biểu đồ hình 10 và kết quả so sánh ở bảng 4 ta thấy năng suất làm việc trên của máy uốn, hàn vòng thép dây tự động sau cải tiến so với máy cũ có kết quả tốt hơn. Cụ thể là năng suất cao hơn $5 \%$, mức tiêu thụ năng lượng giảm $7 \%$ và mức độ hư hỏng giảm $6 \%$.

\section{KẾT LUẬN}

Trong nghiên cứu này, tìm được một phương trình về việc điều chỉnh các thông số ảnh hưởng đến độ loe mối hàn trên sản phẩm đã được đề xuất. Trên cơ sở các kết quả nghiên cứu thực nghiệm đã xác định được các phương trình và biểu diễn mối quan hệ giữa các yếu tố ảnh hưởng đến yêu cầu kĩ thuật của máy uốn, hàn vòng thép dây tự động và nghiên cứu đã chỉ ra rằng các yếu tố ảnh hưởng chính đến độ loe mối hàn trên sản phẩm chủ yếu liên quan đến chế độ hàn theo thứ tự giảm dần là dòng điện, thời gian và lực ép hàn. Kết quả cho thấy máy uốn, hàn vòng thép dây tự động cho năng suất cao gấp 5.8 lần/ nguyên công và giảm $20 \%$ mức tiêu hao năng lượng so với phương pháp uốn, hàn vòng truyền thống và năng suất làm việc của máy hàn vòng thép dây tự động sau cải tiến so với máy cũ cao hơn $5 \%$, mức tiêu thụ năng lượng giảm $7 \%$ và mức độ hư hỏng giảm $6 \%$ và chất lượng mối hàn ổn định hơn.

\section{TÀI LIỆU THAM KHẢO}

[1] M. Pouranvari and S. P. H. Marashi. Factors afecting mechanical properties of resistance spot welds. Materials Science and technology, vol 26, 2010.

[2] M.Zhou. A unified approach to assessing the mechanical performance of resistance spot welds. PhD Dissertation, University of Michigan, An Arbor, MI, 2003. 
[3] L. Boriwal et al. Modelling the resistance spot welding of galvanized steel sheets using Fuzzy logic controller. International Journal of Latest Trends in Engineering and Technology (IJLTET), Vol. 5 Issue 3 May 2015.

[4] Anna Runnemalm et al. Automatic Inspection of Spot Welds by Thermography. Journal of nondestructive evaluation, (33), 3, 398-406, 2014.

[5] P. Puschner, M. Klein , G. Kölzer. Fully Automatic Spot Welding System for Application in Automotive Industry. Soldagem \& Inspeção, 20(4):383-390, 2015.

[6] Satoshi NAKAMURA et al. Automatic Control Technology of Welding Machine MAG-II for Onshore Pipelines. Nippon Steel Technical Report No. 92 July 2005.

Ngày nhận bài: 16/10/2017

Ngày chấp nhận đăng: 08/05/2018 